\title{
A new world of heme function
}

\author{
Yoshihiro Kubo ${ }^{1}$
}

Received: 17 April 2020 / Revised: 24 April 2020 / Accepted: 28 April 2020 / Published online: 10 May 2020

(C) Springer-Verlag GmbH Germany, part of Springer Nature 2020

Heme is a well-known molecule critical for the function of life. It binds to globin protein and the complex, hemoglobin, plays an important role as an oxygen career from the lung to other tissues. Heme is also included in cytochrome in the mitochondrial electron transport system which serves for ATP production in the respiratory chain. Myoglobin with heme in the muscles is known to support oxygen storage. Thus, heme is a critical molecule for the life. On the other side, free form of heme is known to be a toxic molecule which leads the production of reactive oxygen species (ROS), causing various damages to cells. To suppress ROS production, free form of heme is rapidly catalyzed by heme oxygenase (HO). As a result, carbon monoxide (CO) is produced which serves as gaseous signaling molecule.

The long-term collaborative research group of Hoshi and Heinemann reported in 2003 their initial striking finding that free form of hemin (heme with ferric iron, $\mathrm{Fe}^{3+}$ ) binds to the CXXCH motif and inhibits the function of $\mathrm{Ca}^{2+}$ - and voltagegated $\mathrm{K}^{+}$channel, Slo1 [1]. This finding, which showed for the first time a direct link between heme itself and cell excitability, gave strong impact to the research field. They reported also the detailed biophysical background of the inhibition [2].

Some of the voltage-gated $\mathrm{K}^{+}(\mathrm{Kv})$ channels show fast inactivation after activation at depolarized potentials. The molecular mechanism was explained by the "ball-and-chain" model, i.e., plugging of the ion permeating pore occurs from inside by the ball region in the N-terminus cytoplasmic domain, and the inactivation is called N-type inactivation [3]. The authors also reported in 2013 that hemin impairs the Ntype inactivation of voltage-gated $\mathrm{K}^{+}$channel Kv1.4 by direct

This article is a commentary to the original article https://doi.org/10.1007/ s00424-020-02386-1

\section{Yoshihiro Kubo}

ykubo@nips.ac.jp

1 Division of Biophysics and Neurobiology, National Institute for Physiological Sciences, Nishigohnaka38, Myodaiji,

Okazaki, Aichi 444-8585, Japan binding to the CXXH motif in the ball region [4]. This is another striking aspect of direct regulation of ion channel function by heme.

The fast inactivation of $\mathrm{Kv}$ channel is known to be regulated not only by the ball region of the main subunit, but also by the accessary subunit, such as $\operatorname{Kv} \beta$ [5] and dipeptidyl peptidase (DPP) [6]. The research group of Heinemann and Hoshi expanded their scope and newly found that hemin attenuates the inactivation accelerated by $\mathrm{Kv} \beta$ and DPP by direct binding [7]. They first showed the effect of hemin on the N-type "ball" inactivation of Kv3.4 channel. They next presented that the inactivation of Kv1.1 and Kv1.4 by $\mathrm{Kv} \beta 1$ is also decelerated by hemin. Finally they showed the inactivation of Kv4.2 accelerated by DPP6a was also attenuated by hemin. The experiments were done by a well-controlled system, excised patch recordings using transfected HEK293t cells, in which possible contributions of other cytoplasmic factors are minimized. They also directly demonstrated the binding of hemin to $\mathrm{N}$-terminus peptide of $\mathrm{Kv} \beta 1.1$ by microscale thermophoresis analysis [7]. Thus, their conclusions are undoubtedly supported by lines of solid evidence. It appears there is no ambiguity from the molecular physiological aspects using heterologous expression system.

What are the remaining questions to be elucidated in future? One of the obviously major topics will be the physiological relevance. Pieces of further information are awaited for better understanding. The first question is the actual concentration of the free form of heme or hemin in the cell, as discussed by the authors. The effective dose of hemin reported here is relatively low, but we still do not know how high or low or how dynamic it is in various types of cells. A trustable evaluation by direct/ indirect measurement is awaited. The second one is whether or not the direct binding effect is the major effect of heme to the cell function in vivo. At least three distinct effects could be expected to take place by a free form of heme in the cell, i.e., effects of heme itself as reported here, toxic effects of ROS generated by heme, and signaling effects of CO produced by the degradation of heme by heme oxygenase. It is desired to discriminate the effects of heme, ROS, and 
CO explicitly to approach the physiological relevance. The generation of knock-in mice carrying mutation(s) of identified critical cys residue(s) of Slo1 [1], Kv [4] or $\mathrm{Kv} \beta$ subunits [7] could provide us with some insight, although cys mutation might also affect other cys-mediated modifications.

\section{References}

1. Tang XD, Xu R, Reynolds MF, Garcia ML, Heinemann SH, Hoshi T (2003) Haem can bind to and inhibit mammalian calcium-dependent Slo1 BK channels. Nature 425:531-535

2. Horrigan FT, Heinemann SH, Hoshi T (2005) Heme regulates allosteric activation of the Slo1 BK channel. J Gen Physiol 126:7-21

3. Hoshi T, Zagotta WN, Aldrich RW (1990) Biophysical and molecular mechanisms of Shaker potassium channel inactivation. Science $250 \cdot 533-538$
4. Sahoo N, Goradia N, Ohlenschläger O, Schönherr R, Friedrich M, Plass W, Kappl R, Hoshi T, Heinemann SH (2013) Heme impairs the ball-and-chain inactivation of potassium channels. Proc Natl Acad Sci U S A 110:E4036-E4044

5. Rettig J, Heinemann SH, Wunder F, Lorra C, Parcej DN, Dolly JO, Pongs O (1994) Inactivation properties of voltage-gated $\mathrm{K}^{+}$channels altered by presence of beta-subunit. Nature 369:289-294

6. Jerng HH, Qian Y, Pfaffinger PJ (2004) Modulation of Kv4.2 channel expression and gating by dipeptidyl peptidase 10 (DPP10). Biophys J 87:2380-2396

7. Coburger I, Yang K, Bernert A, Wiesel E, Sahoo N, Swain SM, Hoshi T, Schonherr R, Heinemann SH (2020) Impact of intracellular hemin on $\mathrm{N}$-type inactivation of voltage-gated $\mathrm{K}^{+}$channels. Pflugers Arch (in press)

Publisher's note Springer Nature remains neutral with regard to jurisdictional claims in published maps and institutional affiliations. 